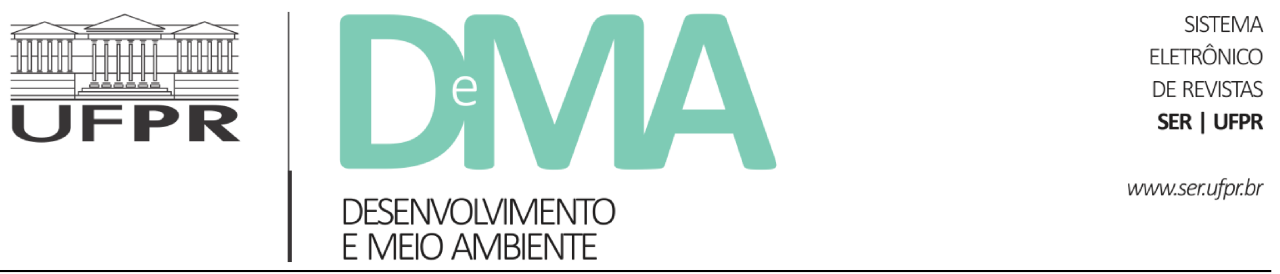

\title{
Estratégias e ações estratégicas nos Planos Municipais da Mata Atlântica: estudo documental em seis municípios
}

\section{Strategy and strategic actions in the Atlantic Forest Municipal Plans: documental study in six municipalities}

\author{
Alexandre de Oliveira e AGUIAR ${ }^{1^{*}}$ \\ ${ }^{1}$ Universidade Nove de Julho (Uninove), São Paulo, SP, Brasil. \\ "E-mail de contato: aaguiar@uni9.pro.br
}

Artigo recebido em 17 de abril de 2018, versão final aceita em 8 de fevereiro de 2019 .

RESUMO: $\quad$ A Mata Atlântica é um dos biomas brasileiros mais ameaçados. Isso é um problema na medida em que os remanescentes desse bioma podem prestar inúmeros serviços ambientais à sociedade. A legislação brasileira permite que municípios elaborem Planos Municipais de Conservação e Recuperação da Mata Atlântica (PMMA), visando a contribuir nesse contexto. O objetivo deste trabalho é estudar as estratégias e ações propostas nos Planos Municipais de Conservação e Recuperação da Mata Atlântica (PMMA) em seis municípios brasileiros. O trabalho é de pesquisa documental e se baseia na análise de conteúdo dos PMMAs nos seis municípios. A análise identificou as categorias de ações e de estratégias a partir dos planos de ação que constam nos documentos. Foram analisadas também características de planejamento como definição de responsáveis, prazos, metas e recursos. Foram identificadas como principais categorias de estratégias: unidades de conservação; arborização urbana; atuação em áreas de preservação permanente; legislação; captação de recursos; educação ambiental; ação política e institucional; ação sobre vetores de pressão; obtenção de novas informações; e economia verde. As ações propostas são, em geral, consistentes com recomendações técnicas e de gestão, no entanto em alguns casos e em alguns temas parecem mais um brainstorming de ideias, sem uma priorização clara, nem definição de responsabilidades, prazos e metas quantitativas. Há necessidade de se trazer um equilíbrio entre a especificidade desses elementos para dar direção e segurança na implantação e flexibilidade que permite adaptar o plano, que tende a ser visto como algo processual e não estático, ao longo do tempo. Ficam como desafios entender as razões para as deficiências no planejamento da implantação, a captação de recursos e o envolvimento de setores produtivos na elaboração e no financiamento das ações.

Palavras-chave: mata atlântica; planejamento estratégico; gestão ambiental municipal.

Desenvolv. Meio Ambiente, v. 50, p. 234-253, abril 2019. 
ABSTRACT: The Atlantic Forest is one of the most threatened Brazilian biomes. This is a problem as the biome's remnants can provide several environmental services to society. Brazilian legislation allows municipalities to elaborate Municipal Plans for Conservation and Recovery of the Atlantic Forest (PMMA), aiming to contribute in this context. This paper aims at studying the strategies and actions proposed in the Municipal Plans for Conservation and Recovery of the Atlantic Forest (PMMA) in six Brazilian municipalities. The study is documentary research and is based on PMMA content analysis in the six municipalities. The analysis identified the categories of actions and strategies from the action plans contained in the documents. Planning characteristics such as definition of managers, deadlines, targets and resources have also been analyzed. The main categories of strategies were identified as: conservation units; urban afforestation; performance in areas of permanent preservation; legislation; fund-raising; environmental education; political and institutional action; action on pressure vectors; obtaining new information; and green economy. The proposed actions are generally consistent with technical and management recommendations, however in some cases and some topics, they seem more a brainstorming of ideas, without clear prioritization, nor definition of responsibilities, deadlines and quantitative targets. There is a need to bring a balance between the specificity of these elements to give direction and security in the deployment, and flexibility that allows adapting the plan, which tends to be seen as procedural rather than static, throughout time. Challenges include understanding the reasons for deficiencies in deployment planning, fundraising, and the involvement of productive sectors in the design and financing of actions.

Keywords: atlantic forest; strategic planning; municipal environmental planning.

\section{Introdução}

A Mata Atlântica é o único bioma brasileiro que tem legislação federal específica para a sua proteção: a Lei Federal 11.428/2006 (Brasil, 2006), conhecida como Lei da Mata Atlântica, regulamentada pelo Decreto Federal 6.660/2008 (Brasil, 2008). A Lei da Mata Atlântica abre a possibilidade de os municípios total ou parcialmente nela inseridos atuarem proativamente na defesa, conservação e recuperação da vegetação nativa desse bioma. O artigo 38 da Lei instituiu o Plano Municipal de Conservação e Recuperação da Mata Atlântica (PMMA), possibilitando acesso a recursos de um fundo específico, que até o momento não foi regulamentado pelo Governo Federal. Desde a publicação desses regulamentos até o ano 2011 não havia tais planos municipais desenvolvidos.
O governo brasileiro, por meio do Ministério do Meio Ambiente, implementou ações visando a promover a conservação e recuperação dos remanescentes da Mata Atlântica por meio do Projeto Mata Atlântica, operado entre 2004 e 2008 com apoio do Banco Mundial, e de 2009 a 2013 com apoio do Governo Federal alemão como Projeto Mata Atlântica II. A fim de fomentar tais planos, o Projeto Mata Atlântica II incluiu, como um de seus componentes, a mobilização e a capacitação de municípios em cada região do país para elaboração dos PMMAs, além da elaboração de um roteiro metodológico para elaboração dos planos (Aguiar \& Steinmetz, 2013).

A possibilidade da implantação pioneira dos PMMAs traz dúvidas e perguntas sobre os desafios que se apresentam: como conseguir a adesão dos municípios a essa política pública federal? Quais os 
melhores métodos para que os planos elaborados sejam consistentes com os objetivos colocados para a conservação e a recuperação da Mata Atlântica? Como trabalhar esse plano no nível local? Quais as dificuldades específicas da administração municipal? Enfim, quais os desafios que surgem na prática?

De acordo com o Observatório dos Municípios envolvidos com os PMMAs, as seis primeiras cidades com PMMAs elaborados, aprovados pelos respectivos Conselhos Municipais de Meio Ambiente e que declararam o PMMA como "em implantação" em 2014 foram: João Pessoa (PB), Maringá (PR), Caxias do Sul (RS), Porto Seguro (BA), Ilhéus (BA) e Sorocaba (SP) (Ambiental Consulting, 2014).

O objetivo deste trabalho é estudar as estratégias e ações propostas nos PMMAs desses municípios, buscando identificar as principais linhas de ação e discutir a consistência quanto aos aspectos de planejamento e os desafios para a implantação dos PMMAs.

O estudo é basicamente documental e se debruçou sobre os PMMAs aprovados nos seis municípios, em particular as ações e estratégias propostas. A análise de conteúdo (Bardin, 2016) foi direcionada à categorização temática das ações, a fim de identificar as principais linhas de ação. A análise das ações concentrou-se nas seções dos documentos que as enumeravam, e não no texto como um todo. A análise resultou num quadro denotando presença ou ausência da ideia em cada PMMA. Além disso, os seguintes temas ligados à implantação foram observados nos documentos: fontes de financiamento, participação de instituições nas ações, atribuição de responsabilidade e prazos para ações.

O texto está estruturado da seguinte forma: esta introdução, que apresenta o tema, objetivos e métodos; uma seção sobre a Mata Atlântica, abordando sua importância, os problemas e a legislação envolvida; uma seção sobre a gestão ambiental municipal, sua estrutura e seus desafios; uma seção que descreve o contexto dos seis PMMAs estudados, uma seção sobre os resultados e que também inclui diálogo com a literatura; e, por fim, uma seção com as conclusões do trabalho.

\section{A Mata Atlântica}

O domínio original de Mata Atlântica no Brasil envolvia cerca de $1.300 .000 \mathrm{~km} 2$, e a floresta se destribuí por 17 estados. Atualmente, a vegetação nativa está reduzida a cerca de $8,5 \%$ da extensão original, considerando-se apenas os fragmentos bem conservados acima de 100 ha (Fundação SOS Mata Atlântica, 2013). Sua área original abrange, total ou parcialmente, mais de 3.400 municípios em que habitam cerca de 120 milhões de brasileiros. Estima-se que na Mata Attântica existam cerca de 20.000 espécies de plantas, e estão catalogadas 849 espécies de aves, 370 de anfíbios, 270 de mamíferos e 350 de peixes (MMA, 2010).

A população que vive no domínio do bioma Mata Atlântica depende da conservação de seus remanescentes para garantir os recursos hídricos, a regulação do clima, a fertilidade do solo, entre outros serviços ambientais. Custos de tratamento de águas provenientes de fontes protegidas por vegetação são menores (MMA, 2010). Além do provimento de água em qualidade e quantidade, Becker \& Seehusen (2011) destacam a polinização natural de plantações, a proteção contra pragas agrícolas e a proteção contra erosão como alguns dos serviços ambientais das áreas de remanescentes 
da Mata Atlântica. Di Stasi et al. (2002), estudando apenas a área do Vale do Ribeira, onde se localizam aproximadamente $70 \%$ dos remanescentes de Mata Atlântica do estado de São Paulo, identificaram 114 espécies de plantas com uso medicinal. Além disso, a região de Mata Atlântica abriga belíssimas paisagens, de valor cênico imensurável, cuja proteção é essencial ao desenvolvimento do ecoturismo (Ambiental Consulting, 2004).

Uma das questões mais importantes na conservação e recuperação da Mata Atlântica é a fragmentação. Com base em diversas fontes bibliográficas, Pardini et al. (2005) destacam que a fragmentação tem um papel determinante na perda de biodiversidade, ou seja, fragmentos menores perdem biodiversidade mais rapidamente. Dixo et al. (2009) confirmaram resultados a esse respeito. Também em áreas em que a floresta está muito fragmentada, as mudanças na estrutura nas suas bordas são mais pronunciadas, intensificando os impactos da ação humana. Destaques similares são feitos por Martensen et al. (2008). Sendo assim, a conexão entre fragmentos tem um potencial importante para contribuir na preservação dos remanescentes, conforme destacam Gálvez et al. (2013). Pütz et al. (2011) demonstram que fragmentos menores tendem a ser direcionados a estágios iniciais de sucessão da floresta. Nesse sentido surgem propostas para criação de corredores entre fragmentos, inclusive entre cidades, por exemplo, como propõem Silva et al. (2017) em cidades do estado de Minas Gerais.

A legislação que protege a Mata Atlântica no Brasil é abrangente e envolve leis relativas à vegetação em geral e à Mata Atlântica especificamente. A lei mais geral é o Código Florestal (Lei Federal 12.651/2012), que define, entre outros elementos importantes, as Áreas de Preservação Permanente
(APP) - áreas em que não se deve intervir, preservando totalmente a vegetação, e a Reserva Legal, uma proporção de área que toda propriedade rural deve observar sem corte de vegetação. Além disso, existe a Lei 9.985/2000, que define os tipos e usos das unidades de conservação. As áreas de conservação são áreas protegidas, em diversos graus de proteção e permissão para intervenção. Elas têm um papel essencial na proteção da biodiversidade e têm também potencial para contribuir em temas econômicos e sociais (Watson et al., 2014).

$O$ processo de supressão de vegetação é regulamentado por estados e municípios com base na legislação federal. Em certos casos a supressão de vegetação é permitida mediante a compensação, ou seja, o empreendedor deve plantar ou prover recursos para plantar certo número de mudas para cada árvore suprimida.

Existe ainda Lei Federal 11.428/2006 - Lei da Mata Atlântica - que define, entre os instrumentos de gestão a serem aplicados, os Planos Municipais de Conservação e Recuperação da Mata Atlântica (PMMA). Esses planos podem ser elaborados no âmbito dos municípios e, sendo aprovados pelo Conselho Municipal de Meio Ambiente, permitem acesso a fundos para a implantação de projetos.

A lei foi regulamentada por um decreto que, entre outros aspectos, estabelece os elementos mínimos dos Planos Municipais da Mata Atlântica:

I - diagnóstico da vegetação nativa contendo mapeamento dos remanescentes em escala de 1:50.000 ou maior;

II - indicação dos principais vetores de desmatamento ou destruição da vegetação nativa;

III - indicação de áreas prioritárias para conservação e recuperação da vegetação nativa; e 
IV - indicações de ações preventivas aos desmatamentos ou destruição da vegetação nativa e de conservação e utilização sustentável da Mata Atlântica no Município. Parágrafo único. [...], devendo ser aprovado pelo Conselho Municipal de Meio Ambiente (Brasil, 2008, s.p.).

O roteiro metodológico adotado pelo Ministério do Meio Ambiente para elaboração de PMMAs foi publicado em 2013 (Dutra et al., 2013) ${ }^{1}$ e é um guia que pode ser usado pelos municípios como apoio a essa atividade. $\mathrm{O}$ documento reconhece que o planejamento de ações para conservação e recuperação da Mata Atlântica no município deve interagir com e levar em conta planos regionais, estaduais e federais no mesmo tema, bem como com outros planos setoriais tais como plano diretor municipal, lei de zoneamento, planos orçamentários, Agenda 21, entre outros. Entre os fundamentos reconhecidos pelo documento estão: a necessidade do envolvimento no nível do município, tanto de agentes públicos quanto privados; a conscientização e o comprometimento da população e dos representantes da sociedade local; o PMMA entendido como instrumento de planejamento para um processo continuado de conservação e recuperação; o PMMA como instrumento setorial que complementa outros instrumentos de planejamento; a fundamentação em informações confiáveis e atualizadas; e a importância do marco legal (Dutra et al., 2013).

O roteiro estabelece quatro etapas básicas para elaboração do PMMA:
Etapa 1: Organização do processo de elaboração

- Criação de equipe de coordenação local;

- Preparação do plano de trabalho;

- Identificação e mobilização dos atores sociais.

\section{Etapa 2: Elaboração do PMMA}

- Diagnóstico de situação atual, com análise de fatores internos e externos;

- Definição de cenários futuros, com construção de cenários alternativos e escolha de um cenário desejado;

- Formulação de plano de ação, com identificação de estratégia e priorização de ações.

\section{Etapa 3: Aprovação do PMMA}

- Aprovação pelo Conselho Municipal de Meio Ambiente;

- Aprovação como outras formas legislativas (lei ou decreto), se desejado pela administração municipal.

\section{Etapa 4: Implementação do PMMA}

- Detalhamento e execução das ações;

- Monitoramento e avaliação dos resultados.

Aguiar \& Steinmetz (2013), com base em um processo de mobilização e capacitação de agentes para elaboração do PMMA, buscaram identificar a percepção dos participantes quanto aos temas que eram conteúdos da capacitação e quanto às barreiras para implantar os PMMAs. Eles identificaram que

\footnotetext{
${ }^{1}$ O roteiro metodológico foi revisado posteriormente em 2017 (Brasil, 2017), incorporando experiências de vários municípios. Alguns dos municípios estudados neste trabalho chegaram a utilizar a primeira versão, outros não utilizaram. A versão atualizada incorporou aspectos voltados a mudanças climáticas e serviços ambientais, mas manteve uma abordagem de planejamento estratégico.
} 
os conteúdos apontados como mais difíceis eram o planejamento estratégico e o monitoramento e que as principais barreiras eram aspectos internos das prefeituras como falta de pessoal e falta de integração entre áreas da administração municipal como principais barreiras a implantação do PMMA.

\section{Gestão ambiental municipal e o planejamento estratégico}

Sendo os Planos Municipais da Mata Atlântica o objeto deste trabalho, interessa em especial a gestão ambiental municipal, e a capacidade dos municípios de liderar e consolidar os PMMAs.

No Brasil, os municípios participam na gestão ambiental. A Constituição (Brasil, 1988) estabelece autonomia para os municípios atuarem na proteção dos recursos naturais. A questão da descentralização da gestão ambiental com maior envolvimento dos municípios não é nova e vem sendo discutida por muitos autores (Maglio, 2000; Salles \& Assunção, 2001; Andion, 2009). Os autores geralmente apontam dificuldades relacionadas à própria estrutura administrativa dos municípios, como falta de recursos humanos e limitações orçamentárias, particularmente nos pequenos municípios. Dados do Instituto Brasileiro de Geografia e Estatística (2012) ilustram essa questão, ao mostrar que menos da metade dos municípios com menos de 5.000 habitantes tem um Conselho de Meio Ambiente, e a proporção cresce continuamente até atingir $100 \%$ dos municípios com mais de 500.000 habitantes.

Somem-se aos fatores estruturais da máquina municipal outros fatores complicadores, tais como a própria complexidade dos problemas ambientais e suas interfaces socioeconômicas, a cultura política estabelecida e a descontinuidade da gestão (Fernandes et al., 2012); a questão da influência sempre presente de fatores locais que pressionam a gestão ambiental (Erol \& Topcu, 2011) e as críticas quanto ao potencial clientelismo e à falta de continuidade da gestão (Lubambo, 2006). Por outro lado, Estevam (2010) discorda que a falta de continuidade da gestão seja necessariamente ruim, dado que as mudanças de rumo podem ser positivas.

Outra questão recorrente é a necessidade de que a gestão ambiental seja democrática e com participação da sociedade, na qual o fluxo de informações seja contínuo e eficaz. As várias formas de participação, comunicação, redes e arranjos institucionais, particularmente os conselhos de meio ambiente, também vêm sendo estudadas por muitos autores (Jacobi, 2003; Milani, 2008; Andion, 2009; Iizuka et al., 2011; Souza \& Novicki, 2011; Iizuka et al., 2012; Nunes et al. 2012). Ao observar os resultados e a discussão que os autores propõem, nota-se que a estrutura de gestão por conselhos de meio ambiente tem seus entusiastas e críticos, mas de forma geral experiências de sucesso são relatadas nos casos em que há um fluxo de comunicação e participação eficaz.

Os temas de gestão estratégica e planejamento estratégico na gestão municipal têm sido discutidos frequentemente. Autores como Rezende \& Ultramari (2007) e Wosniak \& Rezende (2012) ressaltam a importância do planejamento estratégico para a eficácia da administração pública em geral, e municipal em particular. A formação e qualificação dos quadros administrativos como componente essencial da eficácia na gestão pública é trabalhada por autores como Souza (2002) e Oliveira \& Martins (2009). Erol \& Topcu (2011) estudaram um caso na Turquia e propuseram um modelo que inclui o uso 
da ferramenta SWOT (em português Fofa - Forças e Oportunidades, Fraquezas e Ameaças), associada à análise hierárquica de processos. Outros autores também têm sugerido que ferramentas estruturadas associadas ao planejamento estratégico sejam usadas na gestão de florestas em nível local, como Khadka \& Vacik (2012) e Stadt et al. (2006).

Em relação à realidade local, Erol \& Topcu (2011) destacam que as condições locais têm efeitos importantes nas decisões estratégicas sobre gestão de recursos naturais, incluindo os florestais. Sewall et al. (2011) reforçam também a necessidade de levar em conta a realidade local no planejamento da gestão florestal.

Entre as condições locais importantes está o contexto em relação a centralização/descentralização da gestão florestal. Larson \& Ferroukhi (2003), ao estudar diversas experiências de gestão florestal municipal na América Latina, destacam que o processo de descentralização para que as municipalidades passem a gerenciar e tomar as decisões sobre as florestas ainda eram incipientes na época, com resultados variando muito de país para país e mesmo dentro do país. Destacam também que alguns fatores que influenciam a dinâmica da descentralização são fatores socioeconômicos, tamanho da população, grau de urbanização e as características agroecológicas dos municípios. Entre os fatores que podem contribuir para um melhor resultado estão a maior transparência no relato dos resultados para a população local, a existência de alguma forma de agenda de desenvolvimento sustentável e a presença de organizações não governamentais para suporte aos projetos. Por outro lado, a existência de conflitos sociais, a propriedade privada das florestas e a resistência dos governos centrais em abrir mão de poder são barreiras importantes.
As diferentes abordagens do planejamento estratégico são bastante dependentes do conceito utilizado de estratégia. Villar et al. (2017) categorizam as vertentes em clássica, com uma concepção mais racional, em que o processo de planejamento é analítico e o protagonista é o executivo principal; evolucionária, em que o conceito é fatalista e o processo é de adaptação ao ambiente, que é o protagonista do processo; processual, em que o conceito é pragmático, o processo é gradual, contínuo e adaptável, e o protagonista é a "organização"; a vertente sistêmica, em que o processo varia conforme o contexto, num conceito relativista, em que o protagonista é o contexto sociológico; e a estratégia como prática, em que o protagonista é o estrategista e a abordagem é mais social. Embora esses conceitos sejam estudados originariamente em relação a empresas, podem ser utilizados também para analisar organizações públicas ou privadas sem fins lucrativos, mas que têm um propósito claro, como é o caso das prefeituras ou os órgãos que fazem gestão ambiental.

\section{Os primeiros PMMAs}

$\mathrm{Na}$ época em que o projeto de pesquisa se iniciou no ano de 2014, de acordo com o observatório dos municípios envolvidos com PMMAs (Ambiental Consulting, 2014), eram seis as cidades em que tinham sido elaborados os PMMAs e em que o PMMA foi declarado "em implantação" no observatório. A Tabela 1 resume alguns fatos desses seis municípios.

Em João Pessoa, o PMMA foi coordenado pela Secretaria Municipal de Meio Ambiente em parceria com a Fundação SOS Mata Atlântica e com 
TABELA 1 - Características gerais dos municípios com PMMAs em estudo.

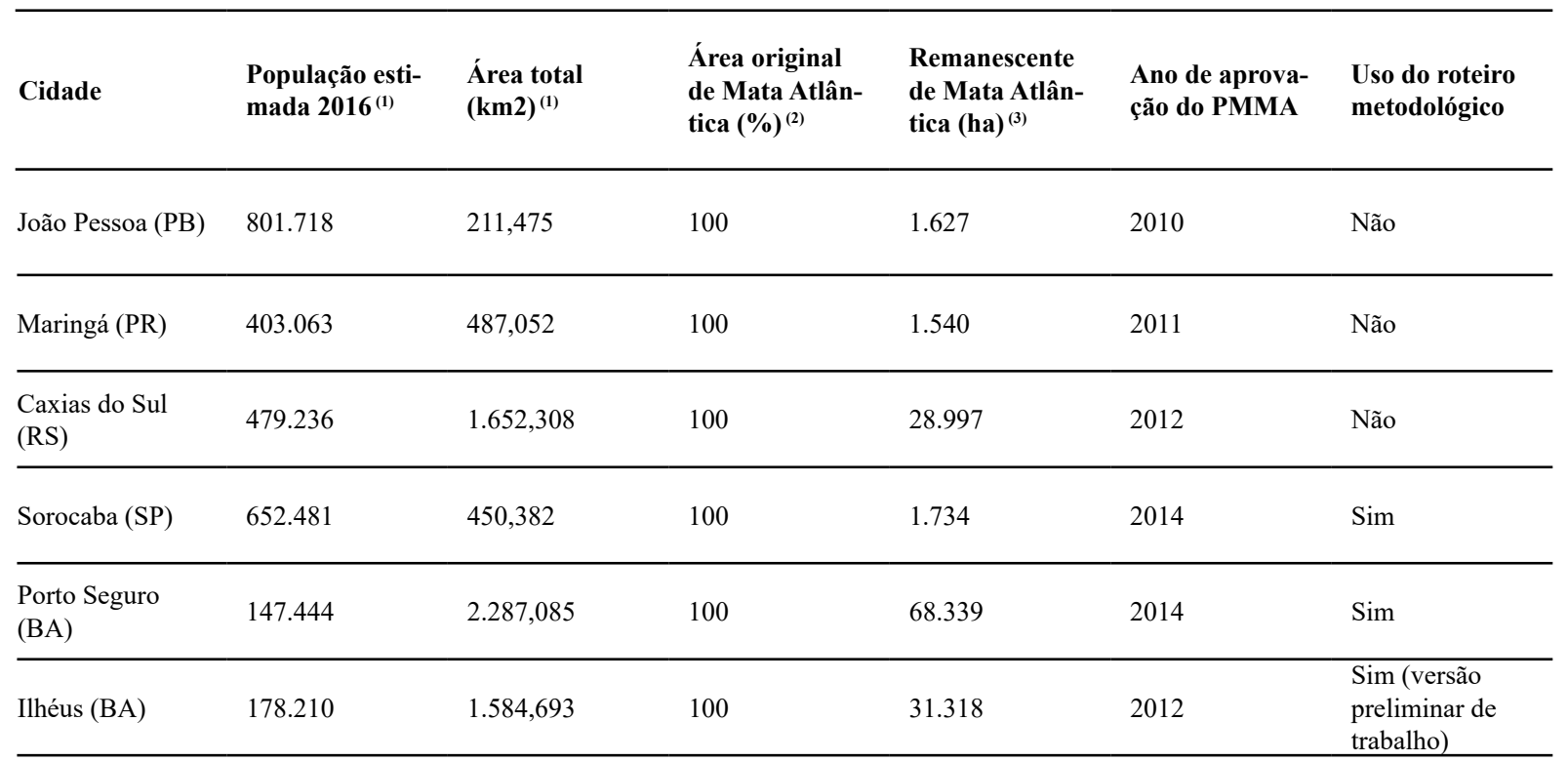

FONTES: (1) Instituto Brasileiro de Geografia e Estatística (2016). (2) Fundação SOS Mata Atlântica \& Instituto Brasileiro de Geografia e Estatística (2012). (3) Fundação SOS Mata Atlântica, 2016.

a colaboração da Secretaria de Planejamento e do Conselho Municipal de Meio Ambiente (COMAM). O plano aponta como um dos principais problemas o excessivo espalhamento da cidade, contido a partir de 2008 quando o plano diretor foi revisado e foram estabelecidos os limites entre zona rural e zona urbana. Os programas habitacionais foram apontados como um vetor de pressão importante na história recente do município. Entre as ações previstas estão a recuperação de APPs e áreas de proteção de manancial, inclusive com cercamento; atuação em zonas de interesse social com relocação de população em habitações subnormais; elaboração de planos de manejo de unidades de conservação; e promoção de atividades de ecoturismo e turismo de aventura (João Pessoa, 2010).
Em Maringá (PR) o PMMA foi elaborado pela Secretaria Municipal de Saneamento Básico e Meio Ambiente da Prefeitura Municipal de Maringá, em parceria com a Fundação SOS Mata Atlântica. O Conselho Municipal de defesa do Meio Ambiente (COMDEMA) também colaborou. O plano apresenta um estudo bastante extenso da vegetação e da fauna no município, bem como dos inúmeros parques e do estágio sucessional da vegetação. Uma das ações mais importantes foi a ampliação, na legislação, das áreas de preservação permanente para 60 metros das margens dos cursos d'água. Há várias ações também visando a promover a conectividade entre os parques da cidade (Maringá, 2012).

O PMMA de Sorocaba foi elaborado pela Secretaria de Meio Ambiente do Município, com 
participação da Universidade Federal de São Carlos. Um dos destaques dos vetores de pressão é a instalação de novos empreendimentos industriais. O diagnóstico é bastante abrangente, abordando os temas propostos no roteiro metodológico, e inclui informações e interações com o Plano Diretor Municipal, principalmente os reconhecimentos de áreas a preservar já definidas. Uma das estratégias de destaque é a ideia de conectar, via Sorocaba, duas unidades de conservação localizadas em municípios vizinhos (Sorocaba, 2014).

A elaboração do PMMA de Porto Seguro foi coordenada pela Secretaria Municipal de Meio Ambiente e Conservação Internacional Brasil, com apoio do Grupo Ambientalista da Bahia (Gamba), Movimento de Defesa de Porto Seguro (MDPS) e Fundação SOS Mata Atlântica. O plano destaca a rica biodiversidade com muitos endemismos no município e ações para redução de vulnerabilidades a mudanças climáticas. Entre os vetores de pressão, destaca-se a expansão dos equipamentos turísticos, que não aproveitam em sua maioria o potencial para ecoturismo e etnoturismo da cidade. Várias ações propostas pelo plano têm interação necessária com instituições públicas fora da estrutura municipal, tais como Fundação Nacional do Índio (Funai), Instituto do meio Ambiente e Recursos Hídricos (Inema) e Instituto Chico Mendes de Conservação da Biodiversidade (ICMBio) (Porto Seguro, 2014).

O PMMA de Ilhéus teve sua elaboração coordenada pelo Grupo Ambientalista da Bahia (Gamba), com financiamento pelo Ministério do Meio Ambiente. A elaboração foi realizada em parceria com a Prefeitura Municipal de Ilhéus, Conselho Municipal de Defesa do Meio Ambiente (Comdema), Universidade Estadual de Santa Cruz (Uesc) e outras organizações. Duas perspectivas de desenvol- vimento se desenham, sendo uma atrelada ao projeto do Complexo Intermodal Porto Sul, que inclui um porto público, terminal privado, a possibilidade de um aeroporto e uma base siderúrgica, além de uma ferrovia, com uma maior pressão por desmatamento; e a outra perspectiva atrelada a uma potencial convivência mais harmônica do desenvolvimento com base no turismo e cultura cacaueira, tendência que vinha sendo predominante e na avaliação do diagnóstico do PMMA não vinha tendo sucesso em promover o desenvolvimento. Entre as ações, estão como exemplos a elaboração de planos de bacias hidrográficas e a revisão do Plano Diretor da cidade (Gamba, 2014).

No município de Caxias do Sul, a Secretaria Municipal de Meio Ambiente elaborou o PMMA com apoio da Fundação SOS Mata Atlântica, Associação Nacional dos Municípios e Meio Ambiente (Anamma) e do Ministério do Meio Ambiente, com a particularidade de ter previsto etapas de diagnóstico (no ano de 2012), experimentação (nos anos de 2013 e 2014) e implantação subsequente. O documento destaca o papel da expansão agrícola e da silvicultura de espécies exóticas como pressões por desmatamento e pela qualidade da biodiversidade, bem como o avanço da ocupação urbana. Destaca-se como ação a aceleração do Cadastro Ambiental Rural, em princípio uma obrigação estadual, com recursos do próprio município (Caxias do Sul, 2012).

Em relação à forma de elaboração dos planos, nota-se que, de maneira geral, a Secretaria de Meio Ambiente geralmente coordenou a maioria dos processos de elaboração do PMMA, à exceção de Ilhéus em que uma instituição externa ficou responsável. Nota-se um aparente predomínio da presença do corpo técnico da prefeitura e de uma instituição técnica externa, seja ONG ou universidade. 


\section{Ações e estratégias}

Uma análise do conteúdo dos PMMAs dos seis municípios foi realizada a fim de identificar estratégias e ações. É importante notar que a nomenclatura do que sejam ações, objetivos e estratégias varia de plano para plano, por isso decidiu-se categorizar as ações em grupos com objetivos e natureza similares. Comentários gerais sobre os planos estão na Tabela 2.
Cada plano é diferente de acordo com a cidade, sua estrutura e suas características específicas. Algumas características dignas de nota estão incluídas na Tabela 2.

Nessa primeira abordagem a categorização pode ser imperfeita, uma vez que uma mesma ação pode ter efeito, na prática, de forma a contribuir para mais de uma dimensão estratégica. Para este trabalho a escolha foi fazer uma análise mais simplificada. Para conveniência da organização

TABELA 2 - Resumos de comentários gerais sobre cada um dos PMMAs estudados (do autor).

MUNICÍPIO COMENTÁRIOS

\begin{tabular}{llll}
\hline & Concentra ações em criação de unidades de conservação e recuperação de APPs. Não pre- \\
JOÃO PESSOA & vê atuar em arborização urbana nem em revisão de legislação. Economia verde focada em turis- \\
& mo sustentável. Principais desafios são relacionados a zoneamento e empreendimentos urbanos.
\end{tabular}

MARINGÁ

Oplanoapresentaumgrandebrainstormdeaçõesemdiversasfrentes.Emboratenhaficadobastanteabrangente, dápoucaindicaçãodas prioridades. Paraaimplantaçãotrazdificuldades pornão apresentarprazosnemresponsabilidadespela execução. Éoúnicodosmunicípiosqueexplicitamenteexpõeproblemascomumaespéciedeplantainvasoraqueprecisasercombatida.

O PMMA foi estabelecido com três etapas sucessivas: diagnóstico, experimentação e implantação. Aetapa de diagnóstico foi um aprofundamento técnico nas diversas temáticas e apoio às propriedades rurais para inscrição no Cadastro Ambiental Rural. Essa ação estendeu-se para a segunda fase, chamada “experimentação", que incluiu também a criação de uma CAXIAS DO SUL base cartográfica de toda a arborização urbana. Essas ações foram financiadas pelo Fundo Municipal de Meio Ambiente, administrado pelo Conselho Municipal de Defesa do Meio Ambiente (Comdema), alimentado por recursos provenientes de multas e licenciamento. Além disso, a compensação ambiental pode ser feita de maneira pecuniária em rubrica específica, permitindo maior garantia de recursos e autonomia para aplicação em recuperação e replantio de vegetação. Apresenta prazos para execução e, para algumas ações, metas quantitativas. Não aponta responsabilidades pelas ações.

O PMMA inclui diversas ações envolvendo instituições externas à prefeitura, particularmenPORTO SEGU- te devido à importância de comunidades indígenas. Há uma presença forte da preocupação de deRO senvolvimento de negócios sustentáveis, particularmente ligados ao turismo e ao extrativismo. Procura uma interação intensa com temáticas correlatas como tratamento de resíduos e gestão de recursos hídricos. Pouco aborda temas em captação de recursos e em melhoria de informações para o próprio PMMA.

\section{ILHÉUS}

As ações previstas são bastante abrangentes, exceto quanto à melhoria de informações para o próprio PMMA. O plano toma partido na questão do dilema entre o desenvolvimento associado ao projeto portuário e o foco num agronegócio mais sustentável. Destaca-se nas propostas de articulação institucional.

Embora Sorocaba tenha estabelecido também ações envolvendo arborização urbana, mapeamen-

SOROCABA to de nascentes e outras atividades típicas, chama a atenção a estratégia de conectar dois grandes fragmentos que estão fora do município. Aparentemente é o único dos seis PMMAs estudados que inclui uma preocupação desse tipo. O PMMA não inclui ações de fortalecimento político-institucional. 
das informações, foram categorizadas apenas na estratégia mais próxima, mesmo que pudessem ser categorizadas em mais de uma estratégia.

A Tabela 3 mostra as presenças de cada categoria nos planos.

De maneira geral, todos os planos buscaram instrumentos que permitem a interação da questão da Mata Atlântica com as várias questões urbanas. Com poucas exceções, os planos incluíram ao menos uma proposta em cada categoria de ação, indicando que há uma compreensão clara de que o tema da conservação e recuperação da Mata Atlântica é multidimensional. Entre as exceções estão a falta de propostas em arborização urbana em João Pessoa, de educação ambiental em Caxias do Sul, de obtenção de novas informações em Porto Seguro e Ilhéus, de economia verde em Caxias do Sul, Maringá e Ilhéus e de ação institucional em Sorocaba.

As categorias de ações estratégicas mais presentes foram: a recuperação ou revegetação em APPs, presente em todos os municípios; a criação de novas unidades de conservação públicas e o plantio e replantio de arborização urbana, indicando que ainda as ações preferenciais são aquelas diretamente ligadas à vegetação.

Algumas categorias de estratégias mostraram-se mais "adensadas" no sentido de que há pouca variação nas linhas de ação e as linhas estão presentes em vários municípios. É o caso das estratégias em unidades de atuação em APPs, arborização urbana e unidades de conservação.

Por outro lado, certas categorias de estratégias mostraram-se bem mais variadas em suas ações, mostrando uma dispersão maior e uma concentração de ações propostas em apenas um ou dois municípios. É o caso das categorias Economia Verde, Obtenção de Novas Informações e Ação
Político-institucional. Essa dispersão está possivelmente associada ao fato de que esses temas são mais dependentes do contexto. Por exemplo, as alternativas em economia verde como agricultura sustentável ou produtos certificados ou turismo sustentável dependem muito da vocação econômica do município e mesmo da cultura de negócios local. $\mathrm{O}$ mesmo se aplica à ação política e institucional, em que a forma de se obter parcerias e envolver outras instituições depende muito do contexto político local. Esse talvez seja um tema que possa, inclusive, variar ao sabor das mudanças de grupos no governo. A questão da obtenção de novas informações, também bastante dispersa, parece depender das possibilidades atuais da prefeitura, por exemplo, ter ou não dados cartográficos ou flora e fauna nativa com suficiente detalhe, e das possibilidades, como a presença de equipe técnica ou parcerias com universidades.

Embora existam atividades planejadas de fiscalização e ações voltadas a ajustes no zoneamento e uso e ocupação do solo, as ações sobre os vetores de pressão são menos expressivas. Se comparado com as ações diretas sobre a vegetação, as ações sobre vetores de pressão são menos variadas e aparecem com menos frequência nos vários PMMAs. Ações na linha da economia verde, buscando negócios mais sustentáveis, também são raras. As parcerias público-privadas, por exemplo, apareceram apenas no município de Maringá. Isso é resultado, possivelmente, de uma ausência dos agentes econômicos na elaboração dos planos e talvez seja um dos principais desafios em termos de articulação institucional.

Sabe-se que a questão da fragmentação da Mata Atlântica é um tema importante, e algumas das ações estratégicas propostas nos planos vão na direção do objetivo de conectar fragmentos. A co- 
TABELA 3 - Resumo dos temas tratados nos planos de ação dos PMMAs estudados (do autor).

CATEGORIAS

DE

ESTRATÉGIAS

\section{Categorias de ação estratégica}

\begin{tabular}{l}
\hline UNIDADES DE \\
CONSERVAÇÃO \\
\hline ARBORIZAÇÃO \\
URBANA \\
\hline ATUAÇÃO EM \\
APPs
\end{tabular}

Cercamento

Novas UCs públicas

Novas RPPN

Planos de manejo

Criação de corredores ecológicos

Plantio/replantio

Plano de arborização

Viveiros

Base de dados para arborização

Cercamento

Recuperação/revegetação

Outras ações de conservação

Ampliação de APPS

LEGISLAÇÃo Revisão de leis de plano diretor

Revisão de leis de uso e parcelamento do solo

IPTU verde

Outorga onerosa

CAPTACÃO DE Pagamento por serviços ambientais

RECURSOS

Recursos de compensação ambiental (Termos de ajustamento de conduta, licenciamento)

Outros incentivos a Reservas Particulares do Patrimônio Natural (RPPN)

Material didático

EDUCAÇÃO

AMBIENTAL

Capacitação na rede de ensino

Ação na comunidade

Divulgação de pesquisas e estudos

Parcerias entre secretarias municipais

Parcerias público-privadas

Captação de recursos

Interação com outras instituições públicas outros municípios estaduais/federais

AÇÃo POLÍTI- Fortalecimento de redes de informações

CA E INSTITU- Mobilização de comunidade (Organizações não governamentais

CIONAL etc.)

Fortalecimento de espaços de articulação política

Capacitações (conselhos, parceiros)

Gestão compartilhada

Consulta pública

Publicação de relatórios

Fiscalização

Termos de ajustamento de conduta

AÇÃO SOBRE

VETORES DE

PRESSÃO $\begin{array}{cccc}\text { João } & \text { Ma- } & \text { Caxias } & \text { Porto } \\ \text { Pessoa } & \text { ringá } & \text { do Sul } & \text { Seguro }\end{array}$ Ilhéus $\begin{gathered}\text { Soro- } \\ \text { caba }\end{gathered}$

\begin{tabular}{llllll}
$\mathrm{X}$ & $\mathrm{X}$ & & & & \\
$\mathrm{X}$ & $\mathrm{X}$ & $\mathrm{X}$ & & $\mathrm{X}$ & $\mathrm{X}$ \\
& & & & $\mathrm{X}$ & $\mathrm{X}$ \\
$\mathrm{X}$ & $\mathrm{X}$ & & $\mathrm{X}$ & & \\
$\mathrm{X}$ & & & & $\mathrm{X}$ & \\
& $\mathrm{X}$ & $\mathrm{X}$ & $\mathrm{X}$ & $\mathrm{X}$ & $\mathrm{X}$ \\
& & & $\mathrm{X}$ & $\mathrm{X}$ & \\
\hline & & & $\mathrm{X}$ & & \\
\hline & & $\mathrm{X}$ & $\mathrm{X}$ & & \\
$\mathrm{X}$ & $\mathrm{X}$ & & & & \\
$\mathrm{X}$ & $\mathrm{X}$ & $\mathrm{X}$ & $\mathrm{X}$ & $\mathrm{X}$ & $\mathrm{X}$ \\
& & & & $\mathrm{X}$ & \\
$\mathrm{X}$ & $\mathrm{X}$ & & & & \\
$\mathrm{X}$ & & & $\mathrm{X}$ & & \\
$\mathrm{X}$ & $\mathrm{X}$ & & & & \\
$\mathrm{X}$ & $\mathrm{X}$ & & & & \\
\hline & & & & & $\mathrm{X}$ \\
\hline & $\mathrm{X}$ & & & $\mathrm{X}$ & \\
\hline
\end{tabular}

X X

$\mathrm{X}$

X $\quad X \quad X$

$\mathrm{X} \quad \mathrm{X}$

$\mathrm{X} \quad \mathrm{X}$

\begin{tabular}{lll}
\multicolumn{1}{c}{$\mathrm{X}$} & \\
$\mathrm{X}$ & $\mathrm{X}$ & $\mathrm{X}$ \\
\hline & & $\mathrm{X}$ \\
\hline
\end{tabular}

$\mathrm{X}$

$\begin{array}{lll} & \mathrm{X} & \\ \mathrm{X} & \mathrm{X} \\ \mathrm{X} & & \mathrm{X}\end{array}$

\begin{tabular}{|c|c|c|c|c|c|}
\hline & & & \multicolumn{3}{|c|}{$X$} \\
\hline \multirow[t]{6}{*}{$X$} & & $X$ & $X$ & X & \\
\hline & & & & $\mathrm{X}$ & \\
\hline & & $X$ & & $\mathrm{X}$ & \\
\hline & & & $X$ & & \\
\hline & & $X$ & & & \\
\hline & & $\mathrm{X}$ & & & \\
\hline $\mathrm{X}$ & $\mathrm{X}$ & & & $\mathrm{X}$ & \\
\hline $\mathrm{X}$ & $\mathrm{X}$ & & & & \\
\hline \multicolumn{6}{|l|}{$\mathrm{X}$} \\
\hline & & & $\mathrm{X}$ & $\mathrm{X}$ & \\
\hline & & & & $\mathrm{X}$ & \\
\hline & & X & & & $\mathrm{X}$ \\
\hline & & & $\mathrm{X}$ & & \\
\hline
\end{tabular}


TABELA 3 - Resumo dos temas tratados nos planos de ação dos PMMAs estudados (do autor).

\begin{tabular}{|c|c|c|c|c|c|}
\hline \multirow{10}{*}{$\begin{array}{l}\text { OBTENÇÃO DE } \\
\text { NOVAS INFOR- } \\
\text { MAÇÕES }\end{array}$} & $\underline{\text { Sobre áreas degradadas }}$ & $\mathrm{X}$ & & & \\
\hline & Sobre APPs & $\mathrm{X}$ & & & \\
\hline & Sobrerreservas legais & $\mathrm{X}$ & $\mathrm{X}$ & & \\
\hline & Sobre Zonas de Especial Interesse Social (Zeis) & $\mathrm{X}$ & & & \\
\hline & Sobre potencial turístico & & & & \\
\hline & Sobre espécies de fauna e flora nativas & & $\mathrm{X}$ & & $\mathrm{X}$ \\
\hline & Sobre espécies invasoras & & $\mathrm{X}$ & & \\
\hline & Sobre informações cartográficas & & & & $\mathrm{X}$ \\
\hline & Sobre recursos hídricos & & & & \\
\hline & Sistema de informação digitalizado & $\mathrm{X}$ & $\mathrm{X}$ & & $\mathrm{X}$ \\
\hline \multirow{3}{*}{$\begin{array}{l}\text { ECONOMIA } \\
\text { VERDE }\end{array}$} & Produtos conservacionistas & & & $\mathrm{X}$ & $\mathrm{X}$ \\
\hline & Produtos certificados & & & & $\mathrm{X}$ \\
\hline & Produção agroflorestal & & & $\mathrm{X}$ & \\
\hline
\end{tabular}

nexão entre grandes fragmentos protegidos aparece, por exemplo, em Sorocaba, em que o adensamento da arborização urbana e parques pode contribuir para conectar a Floresta Nacional de Ipanema, no município vizinho, Iperó (SP), e a Área de Proteção Ambiental de Itupararanga, também com área em municípios vizinhos. Em Maringá, por outro lado, o objetivo estabelecido foi conectar parques urbanos por meio da recuperação de mata ciliar. Ilhéus reconheceu o potencial de conexão, sobretudo de reservas particulares do patrimônio natural, e incluiu no plano ações para fortalecimento desse tipo de unidade de conservação, mas sem prever incentivos específicos. Outros municípios também reconheceram a importância das conectividades entre fragmentos, mas não chegaram a prever ações específicas.

Após a análise das categorias de estratégias e ações, é importante analisar também o tema da implantação, ou seja, como se planeja colocar em prática as ações. Espera-se, num planejamento, encontrar definição de prazos, responsáveis e recursos para as ações. A Tabela 4 mostra o que foi observado nos planos.

Os PMMAs estudados em geral não apresentam metas quantitativas, o que torna mais difícil a alocação objetiva de recursos, bem como a avaliação dos projetos. O plano de Caxias do Sul apresenta metas quantitativas para algumas ações. O plano de Sorocaba apresenta metas quantitativas para várias das ações. Embora na fase de elaboração os conselhos municipais de meio ambiente tenham sido envolvidos, não aparecem nas ações elementos para reforçar o acompanhamento por parte dos conselhos nos seis municípios estudados.

Quanto aos recursos, todos os planos preveem formas de captação, mas a maioria não os associa especificamente a determinadas ações. Em Sorocaba, por exemplo, são citados o orçamento municipal, o orçamento do Fehidro (fundo ligado à gestão de recursos hídricos) e recursos dos proprietários de imóveis envolvidos em regulamentações. João 
TABELA 4 - Conteúdos de implementação dos PMMAs.

\begin{tabular}{|c|c|c|c|c|c|c|}
\hline & João Pessoa & Maringá & $\begin{array}{l}\text { Caxias do } \\
\text { Sul }\end{array}$ & Porto Seguro & Ilhéus & Sorocaba \\
\hline $\begin{array}{l}\text { Definição de } \\
\text { responsáveis por cada } \\
\text { ação ou estratégia }\end{array}$ & Não & Não & Não & Sim & Não & Não \\
\hline Definição de prazos & Não & Não & Parcial & $\begin{array}{l}\text { Qualitativo (curto/ } \\
\text { médio/longo) }\end{array}$ & Não & Sim \\
\hline $\begin{array}{l}\text { Definição de metas } \\
\text { quantitativas }\end{array}$ & Não & Não & Não & Não & Não & Sim \\
\hline $\begin{array}{l}\text { Definição de origem dos } \\
\text { recursos }\end{array}$ & Não & Parcial & $\begin{array}{l}\text { Fase I não } \\
\text { Fase II sim }\end{array}$ & $\begin{array}{l}\text { Fundo Municipal do } \\
\text { Meio Ambiente }\end{array}$ & Em parte & Sim \\
\hline
\end{tabular}

Pessoa planejou como parte das ações o lançamento de editais públicos e privados, mas não detalha que tipo ou tendo como alvo quais fontes. Em alguns casos há menção genérica a fontes para o plano como um todo, mas não para ações, como recursos de compensação ambiental ou pagamento por serviços ambientais.

Em relação à definição de responsáveis específicos para cada ação ou estratégia, apenas o município de Porto Seguro apresenta definições. Os demais não citam nada específico nos planos.

\section{Discussão}

Um tema interessante é a característica das estratégias propostas e a visão de estratégia que emerge dos estudos. Uma das questões que chama a atenção é que o roteiro metodológico propõe, a um tempo, um processo bastante racional e analítico, numa abordagem mais clássica, ao mesmo tempo em que valoriza a participação de atores sociais e a compreensão do contexto, variando, portanto, entre a abordagem sistêmica e da estratégia como prática, conforme propõem Villar et al. (2017).

As estratégias e ações propostas nos seis PMMAs estudados são em geral coerentes com o recomendado pela literatura e são abrangentes para incluir tanto elementos técnicos, tais como recuperação da APPs, criação de corredores ecológicos e tratamento de dados utilizando bases digitais georreferenciadas, quanto aspectos de gestão envolvendo mobilização, articulação política e obtenção de recursos.

A coerência com as recomendações da literatura se expressa, por exemplo, na estratégia de João Pessoa de criação de áreas protegidas, que podem ter papel importante dos pontos de vista da biodiversidade, da economia e social, conforme destacam Watson et al. (2014). A criação de áreas protegidas particulares como as RPPNs, que também são um instrumento importante (Piasentin \& Góis, 2016), está referenciada nos planos de Maringá, Ilhéus e Sorocaba. Também a conexão entre fragmentos prevista em Maringá, basicamente ligando parques municipais por meio da recomposição da mata ciliar, 
e a conexão de dois grandes fragmentos localizados em municípios vizinhos, proposta para Sorocaba, o que segue, entre outras, a recomendação de Gálvez et al. (2013) e está em consonância com o proposto por Silva et al. (2017) em municípios mineiros.

Quanto à obtenção de recursos, destaca-se que o recurso mais imediato que se consegue enxergar são os provenientes de compensação ambiental, tanto na forma de plantio direto pelo empreendedor quanto pelo repasse de valores para que o município gerencie a destinação dos recursos. Essa parece ser uma forma interessante de enfrentar as dificuldades de estrutura e escassez de recursos enfrentadas por prefeituras, dificuldades apontadas, por exemplo, por Maglio (2000). Nesse sentido, parece fazer falta uma articulação como instituições estaduais para direcionamento do ICMS ecológico, ferramenta existente nos estados de São Paulo, Paraná e Rio Grande do Sul e que faria sentido, portanto, para os municípios de Sorocaba, Maringá e Caxias do Sul. Nesse sentido, é importante observar o resultado de Ferreira et al. (2015), que confirmaram a relação positiva entre ICMS ecológico e recursos aplicados em gestão ambiental no estado do Rio de Janeiro.

Interessante notar que em quatro dos seis municípios o plano prevê o aperfeiçoamento das informações necessárias para o próprio plano, o que é interessante por permitir que ao longo do tempo sejam coletadas informações adicionais para as revisões periódicas e, portanto, melhorar o próprio PMMA ao longo do tempo. Essa característica vem de uma elaboração do PMMA um pouco mais acelerada, em que se aceita a falta ou imperfeição de algumas informações em troca de consolidar o PMMA mais cedo. Isso faz sentido para evitar longos e desgastantes processos de diagnóstico que nunca terminam, e tem a vantagem de colocar a equipe do PMMA em contato com instituições externas, tais como universidades, empresas e outras fontes dos dados por um período maior de tempo, gerando ligações e parecerias mais sólidas. Esse aspecto não está presente nem na legislação, mas precisa ser observado para o sucesso do PMMA e é coerente com a abordagem processual, conforme classificação de Villar et al. (2017), por trazer ao processo características de adaptabilidade e por permitir temas emergentes ao invés de ser deliberado e prescritivo como um processo mais clássico.

Entre os aspectos técnicos, a obtenção de informações cartográficas aperfeiçoadas está explicitada no plano do município de Sorocaba. Além disso, informações mapeadas são necessárias para estudar as pressões, por exemplo por meio do CAR, os recursos hídricos, entre outras. Os documentos não falam, no entanto, em dificuldades relacionadas à tecnologia e à capacitação para uso de ferramentas digitais de mapeamento que as prefeituras por vezes enfrentam, conforme levantaram Aguiar \& Steinmetz (2013). Dado que as ferramentas de mapeamento são importantes (Pires et al., 2016), esse ponto poderia ter um destaque maior nos planos.

Outro aspecto importante a comentar é a aderência dos planos a certos princípios básicos de planejamento. Os planos de João Pessoa, Maringá, Ilhéus, Caxias do Sul e Sorocaba não apresentam detalhes sobre as responsabilidades pelas ações. Implicitamente, isso leva à responsabilidade da Secretaria de Meio Ambiente. Isso dificulta o gerenciamento das responsabilidades, uma vez que sempre há outras instituições que terão participação, atividades essenciais e, portanto, responsabilidades no processo. No entanto, isso permite uma negociação futura das responsabilidades e flexibilizar caso haja mudanças na administração municipal, tanto em 
termos de estrutura quanto em termo das pessoas que ocupam cargos de peso político. O plano de Porto Seguro, nesse sentido, é o mais completo, pois inclui para cada ação uma instituição responsável e lista outras instituições parceiras envolvidas.

Os planos de João Pessoa, Maringá, Ilhéus e Sorocaba não apresentam prazos para as ações, o que não é condizente com os princípios de planejamento estratégico. O plano de Caxias do Sul prevê datas de início e término para a maioria das ações, mas inclui também ações sem data de término. O PMMA de Porto Seguro apresenta prazo de início "curto" ou "médio", mas não prazo de término. Além disso, embora sejam estabelecidos prazos finais, na maioria das vezes não há um estabelecimento claro de relações de precedência, o que pode causar confusão e frustração na etapa de implantação.

A falta de prazos e metas para ações pode denotar um certo medo dos agentes públicos em assumir compromissos que futuramente poderão ser cobrados pela população e pelos órgãos de controle, ou talvez falta de compreensão sobre como estabelecê-los, em linha com os achados de Aguiar $\&$ Steinmetz (2013). Nesse segundo caso teria sido necessária uma capacitação mais efetiva das equipes de elaboração do PMMA.

Do ponto de vista da continuidade da administração, que é um dos problemas apontados na literatura (Fernandes et al., 2012; Lubambo, 2006), não incluir metas no plano dá mais flexibilidade para que o governante da vez estabeleça suas próprias metas, tornando o sistema mais adaptável aos ventos da política, também permitindo uma adaptação mais rápida às descontinuidades. Conforme argumenta Estevam (2010), as descontinuidades não são necessariamente ruins. No caso do PMMA, essa flexibilidade pode ajudar na correção de rotas e na maleabilidade de articulação política, permitindo uma variedade de ações.

\section{Conclusões e considerações finais}

O trabalho concluiu pela categorização de 10 estratégias básicas, entre as quais se destacam o foco em unidades de conservação, arborização urbana e atuação em APPs com as mais frequentes em todos os municípios, e de maneira mais dispersa estratégias com foco em legislação, captação de recursos, educação ambiental, ação e política institucional, ação sobre vetores de pressão, obtenção de novas informações e economia verde.

Conclui-se que as ações propostas apresentadas nos PMMAs estudados são em geral consistentes com recomendações técnicas e de gestão, incluindo aspectos como recuperação das áreas verdes, compatibilização com legislação, captação de recursos, ação política e institucional e educação ambiental, visando à articulação com a sociedade, e ação sobre vetores de pressão. As ideias colocadas fazem sentido quando vistas à luz da literatura e do conhecimento atual.

A inclusão relativamente frequente de aprimoramento de informações como parte do próprio plano indica uma visão de que o PMMA não deve ser algo estático, aproximando os planos da visão processual, ou seja, o planejamento como algo que não se encerra na edição da primeira versão do plano. No entanto, traz como desafio uma permanente insegurança quanto aos rumos do planejamento, pois a cada entrada de nova informação o plano anterior pode perder sentido. Isso também se caracteriza como um desafio para a gestão.

No entanto, observou-se que o planejamento apresenta-se em alguns aspectos incompleto em 
alguns municípios, principalmente no tocante ao compromisso com metas, prazos e com as responsabilidades pela implantação das ações, ou mesmo insegurança pela falta de certas informações. $\mathrm{Na}$ maioria das ações não há prazos claramente estabelecidos, metas nem responsabilidades. A dificuldade com parte do planejamento já tinha sido apontada pela literatura. Isso se caracteriza como um desafio importante em termos de gestão. Pode ser também resultado da incerteza quanto à captação de recursos, uma vez que alguns planos preveem mecanismos, outros contam somente com o orçamento municipal, mas não há valores compromissados nos planos.

Seria interessante que em cada município fosse encontrado um equilíbrio entre a falta de definição desses critérios e metas e prazos tão rígidos que não permitissem a adaptação do plano ao longo do tempo no permanente processo de planejamento.

Seria importante estudar se as deficiências encontradas no planejamento refletem uma deficiência técnica do pessoal envolvido quanto à capacitação para essa atividade, ou se está presente o medo do compromisso, que pode gerar críticas e até punições aos governantes caso não sejam cumpridas metas e prazos, ou se é uma decisão deliberada o não estabelecimento de metas e prazos para que o andamento da implantação seja adaptado às novas realidades sociais, políticas e econômicas em caso de mudanças no contexto sócio-político.

Por fim, permanecem os desafios de envolver mais intensamente setores da economia que sejam corresponsáveis pela implantação e também pelo financiamento. Há uma preocupação, nos planos, com a captação de recursos.

\section{Agradecimentos}

O presente trabalho foi realizado com apoio do $\mathrm{CNPq}$, Conselho Nacional de Desenvolvimento Científico e Tecnológico - Brasil (processo 471720/2014-3), e suporte do Fundo de Apoio à Pesquisa FAP/UNINOVE.

\section{Referências}

Aguiar, A. O.; Steinmetz, S. Planos Municipais de Conservação e Recuperação da Mata Atlântica: Lições aprendidas num projeto de mobilização e capacitação. In: XXXVII EnANPAD, 2013, Rio de Janeiro - RJ. XXXVII EnANPAD. Rio de Janeiro - RJ: ANPAD, 2013. p. s.p.

Ambiental Consulting. Animais da Mata Atlântica: Patrimônio Natural do Brasil. São Paulo, 2004.

Ambiental Consunting. Observatório dos municípios que estão em processo PMMA. 2014. Disponível em: http:// www.pmma.etc.br/index.php?option=com_content\&view=article\&id=192\&Itemid=1081 Acesso em: out. 2014 .

Andion, C. Contribuições do debate sobre desenvolvimento territorial sustentável para a efetividade da gestão municipal. Política \& Sociedade, 8(14), 181-210, 2009. doi: 10.5007/2175-7984.2009v8n14p181

Bardin, L. Análise de Conteúdo. São Paulo: Edições 70, 2016. Trad. Luis Antonio Reto, Augusto Pinheiro.

Becker, F.; Seehusen, S. E. (Org.). Pagamentos por Serviços Ambientais na Mata Atlânica: lições aprendidas e desafios. Brasília: MMA, 2011. (Série Biodiversidade, 42). Disponível em: http://www.mma.gov.br/publicacoes/biomas/ category/63-mata-atlantica.

Brasil. Constituição. Constituição da República Federativa do Brasil. Brasília, DF: Senado, 1988.

Brasil. Decreto No 6.660, de 21 de novembro de 2008. Regulamenta dispositivos da Lei $n^{\circ} 11.428$, de 22 de dezembro de 2006, que dispõe sobre a utilização e proteção da vegetação nativa do Bioma Mata Atlântica. 
Brasil. Lei $N^{o} 11.428$, de 22 de dezembro de 2006. Dispõe sobre a utilização e proteção da vegetação nativa do Bioma Mata Atlântica, e dá outras providências.

Brasil. Ministério do Meio Ambiente. Roteiro para a elaboração e implementação dos planos municipais de conservação e recuperação da Mata Atlântica. Brasília, DF: MMA, 2017.

Caxias do Sul (município). Plano Municipal de Conservação e Recuperação da Mata Atlântica de Caxias do Sul. 2012. Disponível em: http://pmma.etc.br/index.php?option=com jdownloads \&Itemid $=0$ \& view $=$ viewcategory \& catid $=\overline{7}$. Acesso em: fev. 2015.

Di Stasi, L. C. et al. Medicinal plants popularly used in the Brazilian Tropical Atlantic Forest. Fitoterapia, 73(1), 69-91, 2002. doi: $10.1016 / \mathrm{S} 0367-326 \mathrm{X}(01) 00362-8$

Dixo, M.; Metzger, J. P.; Morgante, J. S.; Zamudio, K. R. Habitat fragmentation reduces genetic diversity and connectivity among toad populations in the Brazilian Atlantic Coastal Forest. Biological Conservation, 142, 1560-1569, 2009. doi: 10.1016/j.biocon.2008.11.016

Dutra, C. M.; Cordeiro, S.; Camargo, H. T.; Cordeiro, L. A.; Deitenbach, A. V. Roteiro para a elaboração dos Planos Municipais de Conservação e Recuperação da Mata Atlântica. Brasília: MMA, 2013

Erol, S. Y.; Topcu, I. An integrated decision aid for identifying and prioritizing strategies in forest management. Environmental Engineering and Management Journal, 10(5), 683-695, 2011. Disponível em: http://www.eemj. icpm.tuiasi.ro/pdfs/vol10/no5/12_142_Erol_10.pdf.

Estevam, D. O. A contínua descontinuidade administrativa e de políticas públicas. In: Seminário das Ciências Sociais Aplicadas, 2. Anais. Criciúma: UNESC, 2010. Disponível em: http://periodicos.unesc.net/index.php/CSA/article/ view/377/385. Acesso em: abr. 2013.

Fernandes, V.; Malheiros, T. F.; Philippi Jr., A.; Sampaio, C. A. C. Metodologia de avaliação estratégica de processo de gestão ambiental municipal. Saúde e Sociedade, 21, supl. 3, 128-143, 2012. doi: 10.1590/S0104-12902012000700011

Ferreira, S. A.; Macedo, M. A. S.; Pimenta, M. M.; Siqueira, J. R. M. Impacto do ICMS ecológico nos investimentos em saneamento e gestão ambiental: análise dos municípios do estado do Rio de Janeiro. Revista de Gestão Ambiental e Sustentabilidade, 4(2), 65-82, 2015. doi: 10.5585/geas.v4i2.222

Fundação SOS Mata Atlântica. Aqui tem mata? Disponível em: http://aquitemmata.org.br/\#/. Acesso em: nov. 2016.

Fundação SOS Mata Atlântica. Nossa causa. Disponível em: http://www.sosma.org.br/nossa-causa/a-mata-atlantica/. Acesso em: out. 2013.

Fundação SOS Mata Atlântica \& Instituto Brasileiro de Geografia e Estatística. Atlas dos Remanescentes Florestais da Mata Atlântica Período 2008-2010. 2012. Disponível em: https://www.sosma.org.br/link/atlas2011-12/ATLAS_ apresentacao_2011_2012_COLETIVA.pdf. Acesso em: abr. 2013.

Gálvez, N.; Hernández, F. H.; Laker, J.; Petipas, H. G. R.; Bonacic, C.; Gimona, A.; Hester, A.; Macdonald, D. W. Forest cover outside protected areas plays an important role in the conservation of the Vulnerable guiña Leopardus guigna. Oryx, 47(2), 251-258, 2013. doi: 10.1017/ S0030605312000099

GAMBA - Grupo Ambientalista da Bahia. Plano Municipal de Conservação e Recuperação da Mata Atlântica de Ilhéus - Bahia. 2014. http://pmma.etc.br/index.php?option $=\mathrm{com} \_$jdownloads \&Itemid $=0 \&$ view $=$ viewcategory \& catid=7. Acesso em: fev. 2015.

Iizuka, E. S.; Gonçalves-Dias, S. L. F.; Aguerre, P. Gestão social e cidadania deliberativa: a experiência de Ilha Comprida - São Paulo. Cadernos Ebape. 9(3), 748-779, 2011.

Iizuka, E. S.; Gonçalves-Dias, S. L. F.; Aguerre, P. Reflexões sobre o desenvolvimento territorial sustentável, gestão social e cidadania deliberativa: o caso da bacia do rio Almada (BA). Revista de Administração Pública, 46(6), 1599-1623, 2012.

IBGE - Instituto Brasileiro de Geografia e Estatística. Perfil dos Municípios Brasileiros 2011. IBGE, 2012. Disponível em: http://www.ibge.gov.br/home/estatistica/economia/ perfilmunic/2011/default.shtm. Acesso em: mai. 2013.

IBGE - Instituto Brasileiro de Geografia e Estatística. Cidades@. Disponível em: http://cidades.ibge.gov.br/xtras/perfil. php?lang=\&codmun=292530\&search=bahia|porto-seguro. 
Acesso em: nov. 2016.

Jacobi, P. R. Espaços públicos e práticas participativas na gestão do meio ambiente no Brasil. Sociedade e Estado, 18(1-2), 315-338, 2003. doi: 10.1590/S010269922003000100015.

João Pessoa (município). Secretaria Municipal de Meio Ambiente. Plano Municipal de Conservação e Recuperação da Mata Atlântica. 2010. Disponível em: http://pmma.etc. br/index.php?option $=$ com_jdownloads\&Itemid $=0 \&$ view $=-$ viewcategory\&catid=7. Acesso em: fev. 2015.

Khadka, C.; Vacik, H. Use of multi-criteria analysis (MCA) for supporting community forest management. iForest, 5(1), 60-71, 2012. doi: 10.3832/ifor0608-009

Larson, A. M.; Ferroukhi, L. Conclusions. In: Ferroukhi, L. (Ed.). Municipal Forest Management in Latin America. Bogor, Indonesia: CIFOR, IDRC, 2003.p. 213-232.

Lubambo, C. W. Desempenho da Gestão Pública: que variáveis compõem a aprovação popular em pequenos municípios? Sociologias, 8(16), 86-125, 2006.

Maglio, I. C. A descentralização da Gestão Ambiental no Brasil: o papel dos órgãos estaduais e a relação com o poder local. São Paulo, Dissertação (Mestrado em Saúde Pública) - USP, 2000. Disponível em: http://www.teses.usp. br/teses/disponiveis/6/6134/tde-01072008-145252/publico/ IvanMaglio.pdf.

Maringá (município). Secretaria de Meio Ambiente (SEMA). Plano Municipal de Conservação e Recuperação da Mata Atlântica - Maringá - Paraná. 2012. Disponível em: http://pmma.etc.br/index.php?option=com_jdownloads $\&$ Itemid $=0 \&$ view $=$ viewcategory $\&$ catid $=7$. Acesso em: fev. 2015.

Martensen, A. C.; Pimentel, R. G; Metzger, J. P. Relative effects of fragment size and connectivity on bird community in the Atlantic Rain Forest: Implications for conservation. Biological Conservation, 141(9), 2184-2192, 2008. doi: 10.1016/j.biocon.2008.06.008

Milani, C. R. S. O princípio da participação social na gestão de políticas públicas locais: uma análise de experiências latino-americanas e europeias. Revista Brasileira de Administração Pública, 42, 551-79, 2008. Disponível em: http:// www.scielo.br/pdf/rap/v42n3/a06v42n3.pdf/

MMA - Ministério do Meio Ambiente. Secretaria de Biodiversidade e Florestas. Núcleo Mata Atlântica e Pampa. Mata Atlântica: patrimônio nacional dos brasileiros. Brasília: MMA, 2010. [organizadores Maura Campanili e Wigold Bertoldo Schaffer]. Disponível em: http://livroaberto.ibict. br/handle/1/984. Acesso em: abr. 2018.

Nunes, M. R.; Philippi Jr., A.; Fernandes, V. A Atuação de conselhos do meio ambiente na gestão ambiental local. Saúde e Sociedade, 21, suppl. 3, 48-60, 2012. Disponível em: https:/www.scielosp.org/pdf/sausoc/v21s3/05.pdf

Oliveira, N.; Martins, C. H. B. Dimensão institucional da sustentabilidade: gestão ambiental em municípios gaúchos. Indicadores Econômicos FEE, 37, 1 s.p., 2009. Disponível em: https://revistas.fee.tche.br/index.php/indicadores/article/view/2219/2616

Pardini, R.; Souza, S. M.; Braga-Neto, R.; Metzger, J. P. The role of forest structure, fragment size and corridors in maintaining small mammal abundance and diversity in an Atlantic forest landscape. Biological Conservation, 124(2), 253-266, 2005. doi: 10.1016/j.biocon.2005.01.033

Piasentin, F. B.; Góis, S. L. Conservação de remanescentes florestais no Brasil: considerações sobre os principais instrumentos de gestão ambiental. Desenvolvimento e Meio Ambiente, 36, 115-134, 2016. doi: 10.5380/dma.v36i0.42518

Pires, V. R. O.; Garcia, M. A.; Martines, M. R.; Toppa, R. H. Mapeamento do uso e ocupação da terra como subsídio para o planejamento ambiental Land use and ocupation mapping as support to environmental planning. Ambiência, 12(3), 899-908, 2016.

Porto Seguro (município). Secretaria Municipal de Meio Ambiente de Porto Seguro. Plano Municipal de Conservação e Recuperação da Mata Atlântica de Porto Seguro - Bahia. 2014. http://pmma.etc.br/index.php?option=com jdownloads \&Itemid $=0 \&$ view $=$ viewcategory \& $\operatorname{catid}=7$. Acesso em: fev. 2015.

Pütz, S.; Groeneveld, J.; Alves, L. F.; Metzger, J. P.; Huth, A. Fragmentation drives tropical forest fragments to early successional states: A modelling study for Brazilian Atlantic forests. Ecological Modelling, 222, 1986-1997, 2011. doi: 10.1016/j.ecolmodel.2011.03.038 
Rezende, D. A.; Ultramari, C. Plano diretor e planejamento estratégico municipal: introdução teórico-conceitual. Revista de Administração Pública, 41(2), 255-271, 2007. Disponível em: http://www.scielo.br/pdf/\%0D/rap/v41n2/05.pdf

Salles, C. P.; Assunção, J. V. Quadro atual e perspectiva da gestão ambiental municipal no Brasil. In: Congresso Brasileiro de Engenharia Sanitária e Ambiental, 21, 2001. Anais. João Pessoa: ABES, 2001. [CD ROM].

Sewall, B. J.; Freestone, A. L.; Moutui, F. E.; Toilibou, N.; Saïd, I.; Toumani, S. M.; Attoumane, D.; Iboura, C. H. Reorienting Systematic Conservation Assessment for Effective Conservation Planning. Conservation Biology, 25(4), 688-696, 2011. doi: 10.1111/j.1523-1739.2011.01697.x

Silva, R. A.; Pereira, J. A. A.; Barros, D. A.; Nascimento, G. O.; Borges, L. A. C. Análise da conectividade estrutural frente às atividades econômicas na mata atlântica: $\mathrm{o}$ caso do quadrilátero ferrífero (Minas Gerais). Revista de Ciências Agroambientais, 15(1), 1-20, 2017. Disponível em: https://periodicos.unemat.br/index.php/rcaa/article/ download/1491/1649

Sorocaba (município). Secretaria do Meio Ambiente. Plano Municipal de Conservação e Recuperação da Mata Atlântica. 2014. Disponível em: http://pmma.etc.br/index. php?option $=$ com jdownloads \&Itemid $=0 \&$ view $=$ viewcategory\&catid=7. Acesso em: 4 fev. 2015.

Souza, D. B.; Novicki, V. A Participação Social na Questão Ambiental: limites e possibilidades nos conselhos de meio ambiente no Brasil. Meta: Avaliação, 3(7), 25-40, 2011. doi: 10.22347/2175-2753v3i7.103

Souza, E. C. L. A capacitação administrativa e a formação de gestores governamentais. Revista de Administração Pública, 36(1), 73-88, 2002. Disponível em: http://bibliotecadigital. fgv.br/ojs/index.php/rap/article/view/6428/5012

Stadt, J. J.; Schieck, J; Stelfox, H. A. Alberta biodiversity monitoring program - monitoring effectiveness of sustainable forest management planning Environmental Monitoring And Assessment, 121, 33-46, 2006. doi: 10.1007/s10661005-9075-7

Villar, E. G.; Walter, S. A.; Braum, L. M. S. Da Estratégia Clássica à Estratégia como Prática: Uma Análise das Concepções de Estratégia e de Estrategistas. Revista Ibero-A- mericana de Estratégia, 16(1), 8-21, 2017. doi: 10.5585/ riae.v16i1.2409

Watson, J. E.; Dudley, N.; Segan, D. B.; Hockings, M. The performance and potential of protected areas. Nature, 515(7525), 67-73, 2014. doi: 10.1038/nature 13947

Wosniak, F. L.; Rezende, D. A. Gestão de estratégias: uma proposta de modelo para os governos locais. Revista de Administração Pública, 46(3), 795-816, 2012. 